\title{
A novel entomological index, Aedes aegypti Breeding Percentage, reveals the geographical spread of the dengue vector in Singapore and serves as a spatial risk indicator for dengue
}

\author{
Janet Ong ${ }^{1}$, Xu Liu', Jayanthi Rajarethinam', Grace Yap ${ }^{1}$, Derek $\mathrm{Ho}^{2}$ and Lee Ching $\mathrm{Ng}^{1,3^{*}}$
}

\begin{abstract}
Background: Aedes aegypti is an efficient primary vector of dengue, and has a heterogeneous distribution in Singapore. Aedes albopictus, a poor vector of dengue, is native and ubiquitous on the island. Though dengue risk follows the dispersal of Ae. aegypti, the spatial distribution of the vector is often poorly characterized. Here, based on the ubiquitous presence of Ae. albopictus, we developed a novel entomological index, Ae. aegypti Breeding Percentage (BP), to demonstrate the expansion of Ae. aegypti into new territories that redefined the dengue burden map in Singapore. We also determined the thresholds of BP that render the specific area higher risk of dengue transmission.

Methods: We performed analysis of dengue fever incidence and Aedes mosquito breeding in Singapore by utilizing island-wide dengue cases and vector surveillance data from 2003 to 2013. The percentage of Ae. aegypti breeding among the total Aedes breeding habitats (BP), and the reported number of dengue fever cases in each year were calculated for each residential grid.

Results: The BP of grids, for every year over the 11-year study period, had a consistent positive correlation with the annual case counts. Our findings suggest that the geographical expansion of Ae. aegypti to previously "non-dengue" areas have contributed substantially to the recent dengue fever incidence in Singapore. Our analysis further indicated that nonendemic areas in Singapore are likely to be at risk of dengue fever outbreaks beyond an Ae. aegypti BP of 20\%.

Conclusions: Our analyses indicate areas with increasing Ae. aegypti BP are likely to become more vulnerable to dengue outbreaks. We propose the usage of Ae. aegypti BP as a factor for spatial risk stratification of dengue fever in endemic countries. The Ae. aegypti BP could be recommended as an indicator for decision making in vector control efforts, and also be used to monitor the geographical expansion of Ae. aegypti.
\end{abstract}

Keywords: Aedes aegypti, Dengue, Entomological index, Spatial risk indicator, Geographical expansion

\footnotetext{
* Correspondence: NG_Lee_Ching@nea.gov.sg

'Environmental Health Institute, National Environment Agency, Singapore,

Singapore

${ }^{3}$ School of Biological Sciences, Nanyang Technological University, Singapore,

Singapore

Full list of author information is available at the end of the article
}

C The Author(s). 2019 Open Access This article is distributed under the terms of the Creative Commons Attribution 4.0 International License (http://creativecommons.org/licenses/by/4.0/), which permits unrestricted use, distribution, and reproduction in any medium, provided you give appropriate credit to the original author(s) and the source, provide a link to the Creative Commons license, and indicate if changes were made. The Creative Commons Public Domain Dedication waiver (http://creativecommons.org/publicdomain/zero/1.0/) applies to the data made available in this article, unless otherwise stated. 


\section{Background}

Dengue fever has caught global attention due to its increasing frequency of major epidemics in recent years. It is estimated that 2.5 billion individuals, residing in more than 100 countries, are at risk of dengue fever, with an annual case burden of approximately 50-100 million infections [1]. Facilitated by the rapid urbanization and increased global travel, dengue fever continues to make its geographical spread across the world.

Dengue fever is caused by the dengue virus (DENV), exists as four different serotypes, DENV1-4. Infection with one serotype confers lifelong immunity only to that particular serotype [2]. Hence, repeated outbreaks in a given population at short intervals are generally due to different serotypes. All four dengue serotypes can be found co-circulating in Singapore at all times [3]. It has been observed previously that there is a cyclical pattern of outbreaks that peaks every five to seven years in Singapore $[4,5]$.

Dengue fever is transmitted to humans by Aedes spp. mosquitoes, mainly by Ae. aegypti. Aedes albopictus has also been incriminated as an important vector of DENV. In Hawaii, parts of China, Seychelles, and more recently, France, where Ae. aegypti was absent or had limited presence, Ae. albopictus was identified as the main vector in the dengue outbreaks [6, 7]. However, reported transmission due to Ae. albopictus is typically less intense and short-lived [8].

In Singapore, the vector status of Ae. aegypti and Ae. albopictus reflects the global situation. Dengue transmission, as indicated by a cluster of cases located within a $150 \mathrm{~m}$ radius and with onset of illness within a 14-day period, co-locates with presence of Ae aegypti [9, 10]. There is no evidence of transmission in places with only Ae. albopictus. The vector status of the two Aedes mosquitoes is corroborated by vector surveillance studies using Gravitraps, which found that mosquitoes caught in areas with clusters of dengue cases were predominantly Ae. aegypti [11]. Potentially infective Aedes, as demonstrated by the presence of dengue viruses in the head of the mosquito, were found to be Ae. aegypti [12]. Aedes aegypti, found in urbanized built-up areas, is thus the primary vector of DENV in the country. Aedes aegypti is known to originate from Africa and was introduced into the coastal cities of South East Asia around 19th century via the shipping industry [13]. The precise time of its arrival to Singapore is not known. In contrast, Ae. albopictus is native and ubiquitous throughout Singapore, due in part to the abundant greenery that lines the streets and adorns the housing estates.

Despite a low Aedes house index of around 2\%, Singapore continues to experience regular outbreaks [14]. In 2005, 2007 and 2013, Singapore experienced explosive dengue fever outbreaks that resulted in 14,032, 8287 and 22,170 indigenous cases, respectively, with incidence rates of 322.5,
180.6 , and 404.9 per 100,000 population [15-17]. All three outbreaks were associated with the replacement of predominant DENV serotypes, which was believed to have played an important role in the escalating number of cases $[15,18]$.

Factors that may have contributed to the population's sensitivity to outbreaks include: (i) rapid increase in population, which grew from 2.1 million in 1970 to 5.4 million by 2013; (ii) rapid urbanization with an extensive transport network of $164 \mathrm{~km}$ of expressway and $199.6 \mathrm{~km}$ of mass rapid transit (MRT) lines across the $720 \mathrm{~km}^{2}$ island; (iii) increased globalization as demonstrated by 26.5 million air arrivals in 2013, as compared to 1.7 million recorded in 1970; (iv) low herd immunity, especially among younger generations, due to decades of low local transmission; and (v) presence of cryptic breeding sites which are difficult to identify and henceforth implement vector control [19-27]. Some of these developments have undoubtedly favoured the expansion of Aedes population as well as the frequent importation of new viruses and rapid dispersal of viruses within the country, all of which facilitate human, vector and virus encounters [28-30]. Expansion of Ae. aegypti to historically 'non-dengue' areas in the country has the potential to expose an immunologically naïve human population to DENV, increasing the risk of outbreaks.

Vector surveillance, recommended by the World Health Organization (WHO), is a routine practice in many dengue endemic countries to provide quantifiable measure of fluctuations in magnitude of dengue vector populations [31, 32]. Globally, the most commonly used indices for vector surveillance are House Index (HI), Container Index $(\mathrm{CI})$ and Breteau index (BI). In Singapore, HI has been used for monitoring the Ae. aegypti population in the community. However, Singapore's vector control programme has brought the HI down from about $50 \%$ in the 1960 s to $0.30 \%$ in the 2000s, way below the target levels for $\mathrm{HI}$ set by the WHO $[9,11,33]$. The low Aedes HI has rendered HI insensitive for gauging Ae. aegypti population in the community and hence, $\mathrm{HI}$ is no longer sensitive for dengue risk assessment. This study therefore aims to introduce a novel vector index based on routine inspection data, with higher spatial resolution and better relevance to spatial dengue transmission risk.

\section{Methods}

\section{Data collection and preparation}

Georeferenced data on dengue fever cases and Aedes spp. larval counts from routine surveillance in the main island of Singapore from 2003 to 2013 were extracted from the Geographical Information System (GIS) Database of the National Environment Agency (NEA), Singapore. The daily-updated database is part of the national vector control programme. Dengue is a notifiable disease in Singapore. It is mandatory 
for medical practitioners and clinical laboratories to notify all clinically diagnoses and laboratory-confirmed dengue cases to the Ministry of Health (MOH), Singapore [34]. The laboratory confirmation of clinical diagnoses is achieved through either NS1 antigen detection or viral RNA detection by PCR or IgM detection [35, 36]. The notification information included demographic data, travel history, onset of illness, residential and workplace address. Dengue cases were tagged to the address, either residential or workplace address, after epidemiological investigation had been carried out by officers to determine and confirm the location where the cases acquired dengue. The Aedes larval surveillance data are derived from daily inspection of residential and non-residential premises for mosquito breeding in habitats such as drains, gutters and containers, conducted by approximately 1000 vector control officers. These inspections include those scheduled for regular preventive surveillance, and those conducted in response to dengue transmission in a location. If mosquito breeding was discovered or suspected, a georeferenced sample would be taken and sent to NEA laboratory for species identification. The Aedes larvae were identified by trained taxonomists using morphological keys [37]. A breeding site was defined as a positive Ae. aegypti breeding site if the sample was identified to contain at least one Ae. aegypti larva. We also obtained data of annual total population, resident population and residential dwelling units from reports published by the Singapore Department of Statistics [20,38].

As administrative areas of Singapore are of irregular shapes and varying sizes, we superimposed a $1 \times 1 \mathrm{~km}$ grid system on the map of the main island and used these fixed grid cells as study units to minimize the normalization required. We choose $1 \times 1 \mathrm{~km}$ to balance the need for a relatively large area of each study unit and a large sample size at the same time, given Singapore's size. Grids were categorized into non-residential and residential based on the land use in 2013 as determined by where the centroids of grids fall within. After excluding major industrial and forested areas, 213 residential grids were included in the analyses.

\section{Definition and estimation of Ae. aegypti Breeding Percentage (BP)}

Aedes aegypti Breeding Percentage (BP) was defined as the proportion of Ae. aegypti positive breeding sites of the total number of Aedes spp. positive breeding sites found (Ae. aegypti and Ae. albopictus) in a defined area within a defined time period:

$$
\begin{aligned}
\mathrm{BP}= & (\text { No.of Ae.aegypti positive breeding sites } / \\
& \text { No.of Aedes spp.positive breeding sites }) \times 100
\end{aligned}
$$

BP, expressed as a percentage, assumes the ubiquitous presence of native Ae. albopictus population in Singapore, and uses total Aedes breeding for the normalization of field data in order to cancel out the sampling error from non-systematic inspection and cryptic breeding sites. Only positive breeding sites were taken to avoid inclusion of grids with no data which could arise because the grids have not been visited or checked thoroughly by the field inspectors. This seeks to address non-systematic operational inspection, which tends to bias towards outbreak areas.

To estimate the yearly BP for each grid, we mapped the location of Aedes breeding sites onto each grid and extracted the number of Ae. aegypti and/or Ae. albopictus breeding sites found within each grid, for each year from 2003 to 2013. Due to the fact that breeding data are the result of non-probability sampling, BP estimated by percentage of Ae. aegypti breeding among all Aedes breeding found could incur a large error, especially when the denominator was small. Here, we set the threshold of denominator to be ten, after examining the distribution of the number of Aedes breeding sites found. BP for grids with at least ten Aedes breeding found in a year was calculated by definition. For other grids, the small number of breeding sites gives low confidence, BP were thus estimated using ordinary Kriging with a spherical variogram model (Additional file 1: Figure S1). BP in residential grids were compared graphically year to year to assess the change in geographical distribution of $A e$. aegypti population over the years. Temporal trend of $\mathrm{BP}$ was assessed by the median BP of each year.

\section{Estimation and analysis of case burden}

To estimate yearly dengue case burden for each grid, dengue fever cases from 2003 to 2013 were plotted spatially onto the grids. Case burden in residential grids was plotted as pixel images and compared yearly to assess the change in geographical distribution of dengue transmission over the years. Temporal trend of case burden was assessed by median case count of each year. Some analyses involving case burden used the transformed variant of case count to stabilize the variance across different scales. The transformation used was $f(Y)=\log (Y+1)$ where $Y$ is the grid case count.

\section{Association between BP and case burden}

The spatial relationship between BP and case count was assessed by Spearman's correlation between grid BP and grid case count. The correlation was analyzed separately for each year to check for consistency and robustness of the relationship.

\section{Determination of BP thresholds for risk stratification}

To use BP in novel dengue areas as a risk flag for preventive surveillance, we defined case burden as a categorical variable. For a particular year, a grid was classified as high dengue burden if it contributed at least 
$0.5 \%$ of all cases, moderate dengue burden if it contributed $0.1-0.5 \%$, and low dengue burden if less than $0.1 \%$. For example, if a grid reported less than 22 cases in 2013, it was labelled as low burden area in 2013. Therefore, each grid scored 11 class labels of "high burden", "moderate burden" or "low burden" during the 11-year study period. The accuracy of using BP to discriminate these areas was determined based on the standard values of area under the ROC curve (AUC): AUC $<0.5$ (not discriminative); $0.5<$ AUC $<0.7$ (less discriminative) $0.7<$ AUC $<0.9$ (moderately discriminative); and $0.9<$ AUC $<1.0$ (highly discriminative).

\section{Alignment between increase in BP and increase human population and number of housing units}

The alignment between increase in BP and increase in human population and residential dwelling units was assessed by Spearman's correlation, in attempt to provide an explanation for the increased BP.

\section{Data analysis}

Data and statistical analyses were conducted using $\mathrm{R}$ version 3.1.1 [39]. R packages utilized in the study include sp, gstat, sciplot and pROC [40-43]. All methods used for analyses are described in respective sections. $P$-values of Spearman's correlation tests were computed via the asymptotic t-approximation. A significance level of 0.05 , and with Bonferroni correction in the case of multiple comparisons, was utilized in this study.

\section{Results}

In the present study, we used a novel entomological index, Ae aegypti BP, which has consistent positive spatial correlation with localized dengue fever burden to demonstrate the geographical expansion of Ae. aegypti in Singapore. This simple index uses field entomological data that need not be consistently and systematically collected. Analysis using the BP further revealed that the geographical expansion of Ae. aegypti has contributed to the recent increase and altered corresponding distribution of dengue cases in Singapore.

\section{Association between BP and case burden}

Every geographical grid was tagged with case count and BP for each of the 11 years. BP was consistently positively associated with case count, though the Spearman's correlation coefficient varies across the years, ranging between $0.547-$ 0.737 (Fig. 1). All correlations were significant $(P<0.0001)$ with respect to the significance level of 0.05 .

\section{Determination of BP thresholds for risk stratification for dengue control}

Based on the contribution to national burden of dengue, the grids were stratified into three levels of disease burden and their average BP were calculated yearly. Through the 11 years, each of the high burden grids $(>0.5 \%$ of national annual cases) was characterized by BP of range $40-60 \%$, moderate burden grids $(0.1-0.5 \%$ of national annual cases) were associated with BP of 20-40\%, mostly more than $30 \%$, and low burden grids $(<0.1 \%$ of national annual cases) had BP less than 20\% (Fig. 2). As a result, grids with BP less than 20\% were considered "low risk", BP of 20 $40 \%$ as "moderate risk", and BP above $40 \%$ as "high risk".

Figure 3 shows the ROC curve of using BP to categorize risk of dengue transmission. For classification of low dengue burden versus other categories, the AUC was 0.812 (95\% CI: 0.793-0.831), indicating moderately discriminative capability of BP. Using a low BP as threshold to predict dengue risk was less sensitive and highly specific, whereas using high BP, though highly sensitive, resulted in low specificity. Setting the $20 \%$ BP as a threshold to predict dengue risk resulted in $75.31 \%$ specificity and $75.28 \%$ sensitivity. The 20\% BP was based on the cut-off between the low and moderate burden grids. At higher level of $40 \%$ derived from BP of high burden grids, specificity was $71.69 \%$ and sensitivity was $83.85 \%$.

\section{Spatial and temporal characteristics of BP}

Graphical presentation of BP in 2003 and 2013 (Fig. 4, left panels) showed that in 2003, Ae. aegypti was present in moderate and high proportion ( $\mathrm{BP} \geq 20 \%$ ), mostly in the central and eastern part of Singapore. However, by 2013, grids with $\mathrm{BP} \geq 20 \%$ were found in the western part and north corner. Areas in the central and eastern part also showed higher BP values in 2013. The right panels of Fig. 4 show the corresponding spread of dengue from the central and eastern part of Singapore to the northern and western parts. Of significance are areas that had evolved from low risk to high risk in the last 11 years. These areas include the Jurong and Choa Chu Kang areas around 2004-2005; Clementi, Bukit Batok and Pasir Ris areas around 2006 and 2007; Queenstown and Central areas during 2008-2012; and the Upper Thomson area around 2013.

In general, there has been an increase in the percentage of geographical grids that show moderate or high level of BP (Fig. 5). In 19 of the grids, Ae. aegypti was not found in $2003(\mathrm{BP}=0 \%)$ but was found to have BP ranging between $5-80 \%$ by 2013 . This demonstrates the spatial expansion of Ae aegypti over the 11-year period. At the same time, the median grid BP increased from $15.87 \%$ in 2003 to $42.47 \%$ in 2013 (Fig. 6), indicating a general increasing trend of $\mathrm{BP}$ over time, regardless of lull or outbreak (2005, 2007 and 2013) years.

Alignment between increase in BP and increase in human population and number of housing units

Increase in BP corresponds to increase in human population and number of residential dwelling units (Fig. 7). 

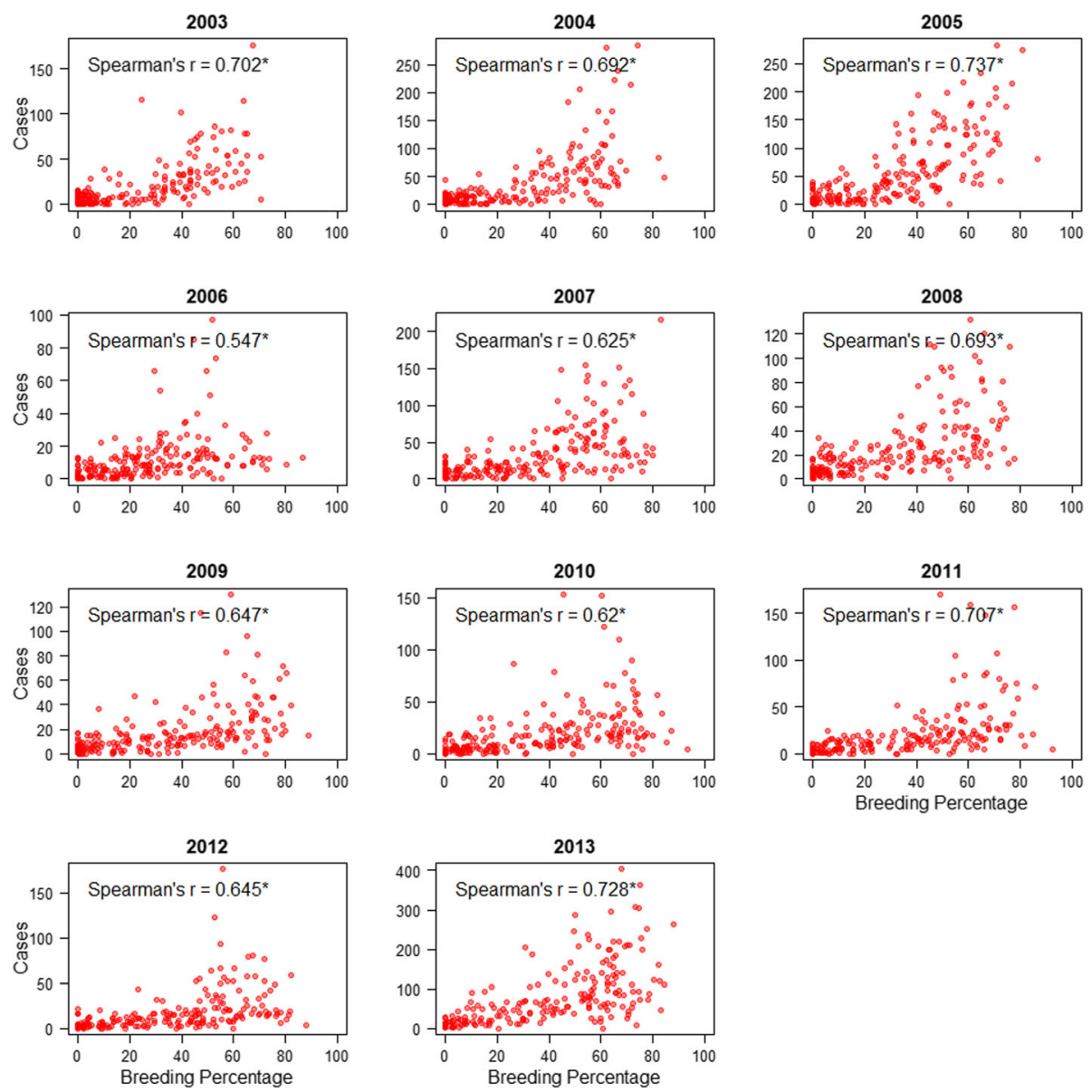

Fig. 1 Relationship between Breeding Percentage (BP) and case count assessed by Spearman's rank correlation test. Asterisks indicate statistical significance

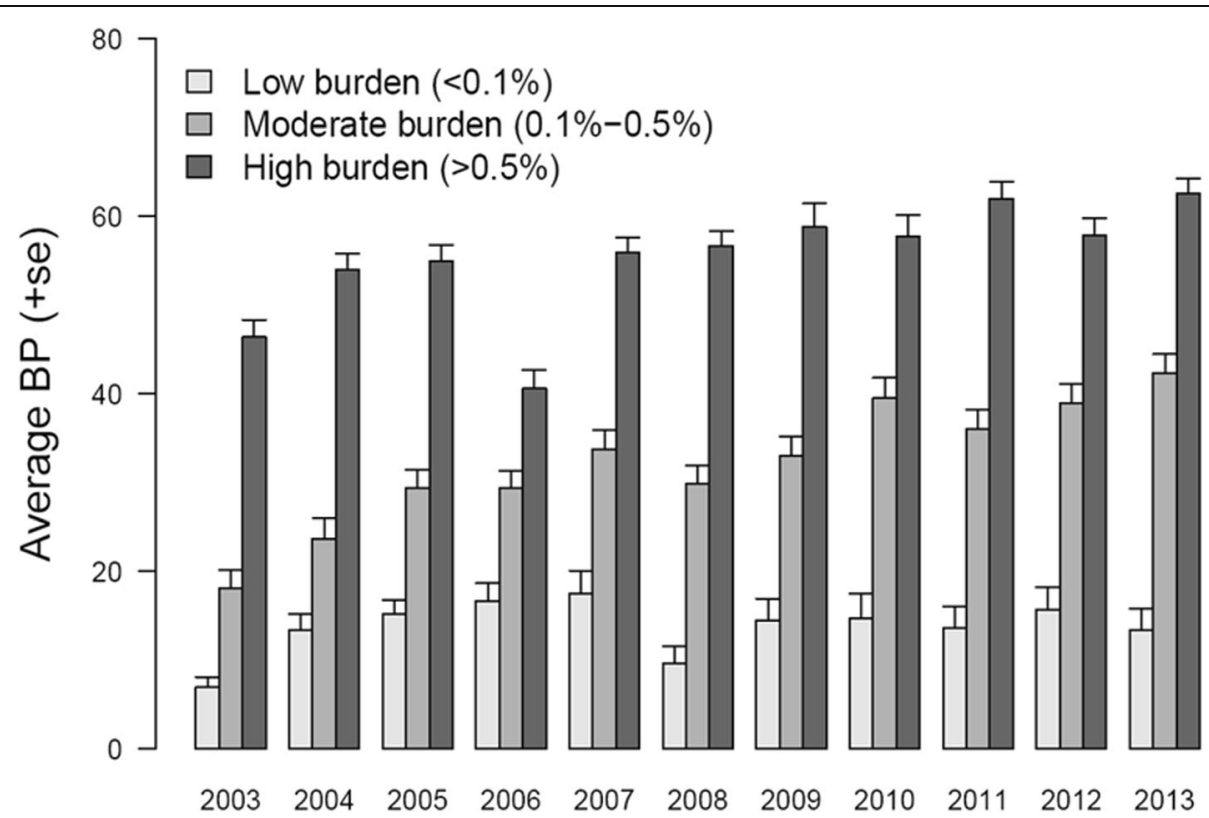

Fig. 2 Differences in Breeding Percentage (BP) between low burden, moderate burden and high burden areas. Average BP for low burden (light grey bars), moderate burden (grey bars) and high burden (dark grey bars) areas are shown with standard error bars 

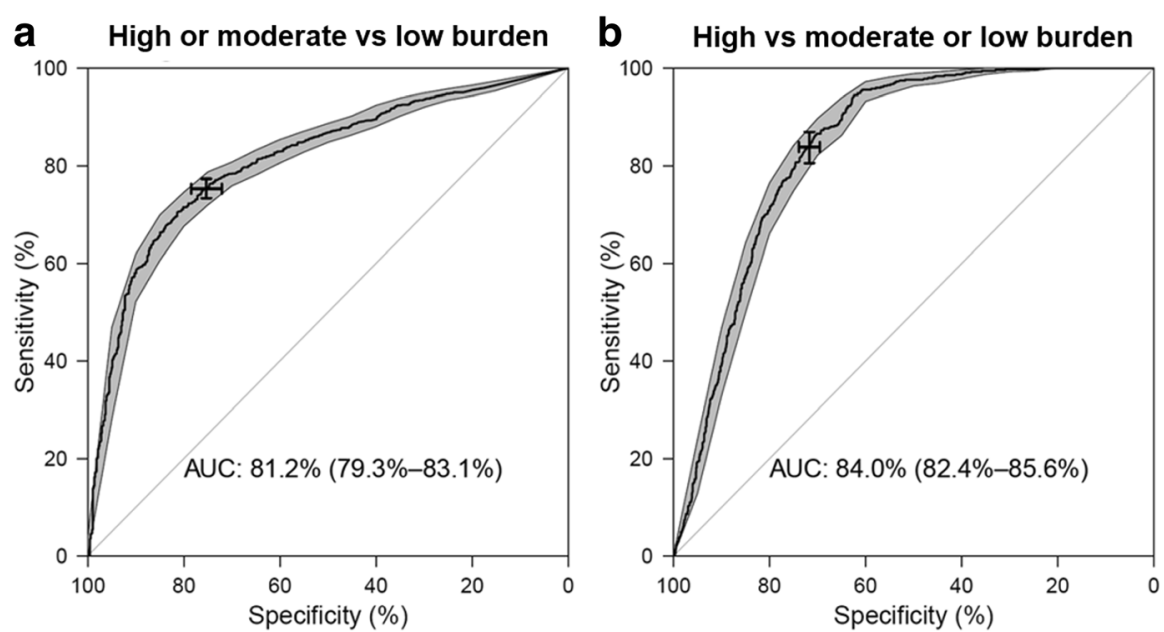

Fig. 3 Receiver operating characteristics curves (black thick line) of classification of areas as "high-burden", "moderate burden" and "low burden" by Breeding Percentage, and the $95 \% \mathrm{Cl}$ (grey shaded area) of sensitivity over a selected set of specificity. a Classification of "high-burden" or "moderate burden" versus "low burden". The area under the curve is 0.812 with $95 \% \mathrm{Cl}: 0.793-0.831$. The sensitivity-specificity combination given by the chosen threshold $20 \%$ is indicated on the curve: sensitivity $=75.28 \%$ (95\% Cl: $73.22-77.22 \%)$; specificity $=75.31 \%$ (95\% Cl: 71.89-78.57\%). b Classification of "highburden" versus "moderate burden" or "low burden". The area under the curve is 0.840 with $95 \%$ Cl: $0.824-0.856$. The sensitivity-specificity combination given by the chosen threshold $40 \%$ is indicated on the curve: sensitivity $=83.85 \%$ (95\% Cl: 80.55-86.97\%); specificity $=71.69 \%$ (95\% Cl: 69.52-73.86\%)

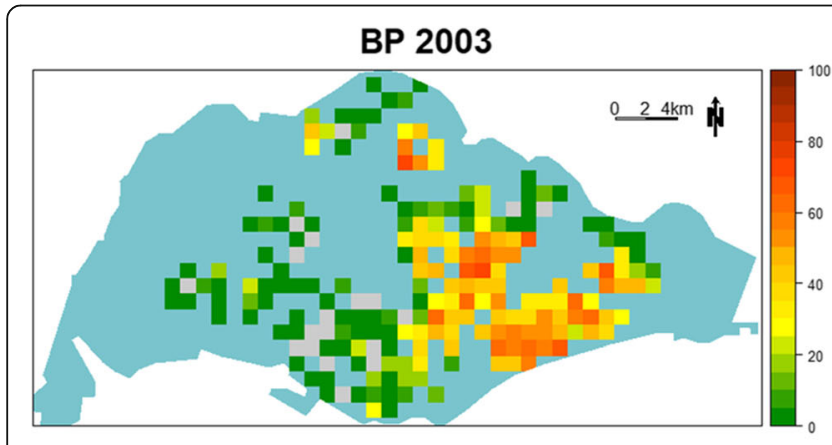

BP 2013

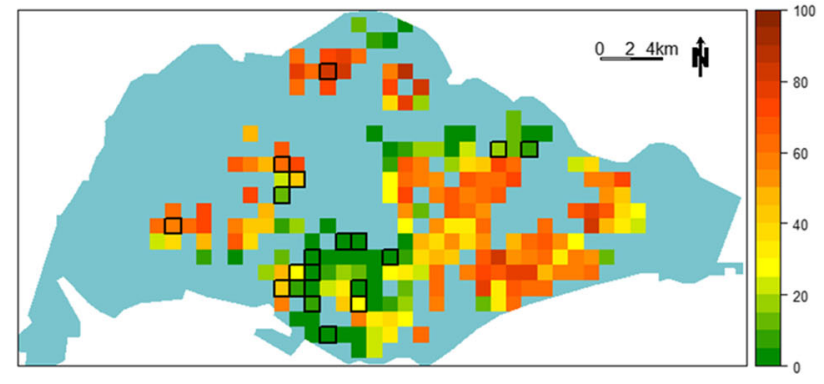

Cases 2003

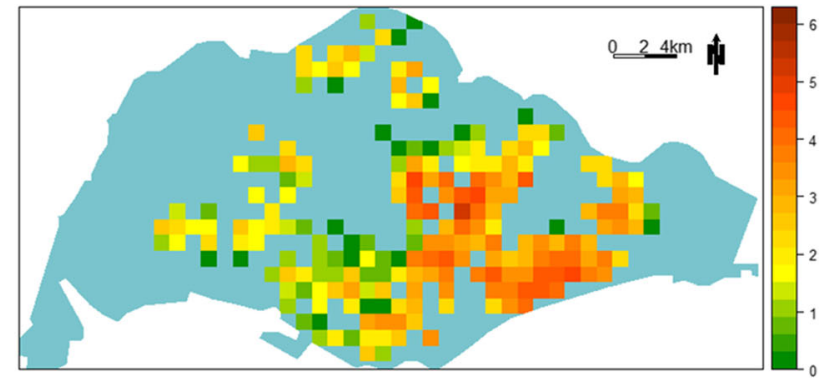

Cases 2013

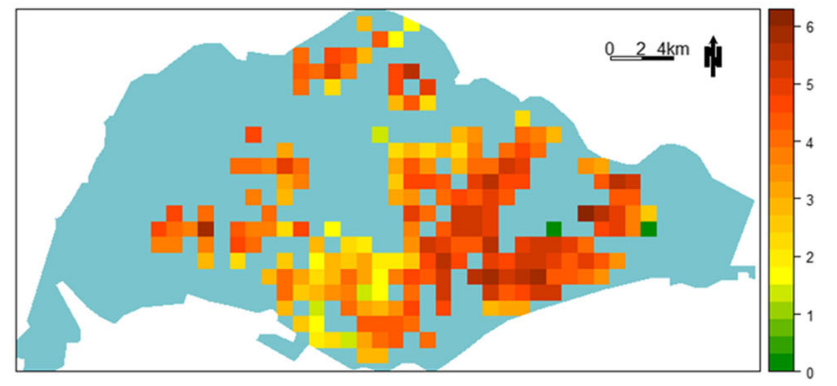

Fig. 4 Spatial distribution of Breeding Percentage (BP) and transformed dengue case burden in 2003 and 2013. Left panels: values of BP are color-coded, with difference shades of green indicating BPs $<20 \%$, yellow shades indicating BPs between $20 \%$ and $40 \%$, and orange through dark red indicating BPs $\geq 40 \%$. Residential grids with BP 0\% in 2003 were highlighted in gray and outlined in black in the 2013 map. In 2003, areas with higher BP were exclusively in the eastern part of the island. By 2013, Ae. aegypti has expanded into the northern and western part of the island. The spatial expansion of Ae. aegypti is also illustrated by areas that turn from a BP of $0 \%$ to having a BP of $>0 \%$. Furthermore, areas that had relatively higher BPs in 2003 also registered higher levels of BPs in 2013, as visualized by the color gradient. Right panels: transformed dengue case burden in residential grids in 2003 and 2013 are represented by colors, with green being the least dengue burden and dark red being the highest burden. Vertical comparisons illustrate the spatial expansion of Ae. aegypti and dengue transmission. Horizontal comparisons reveal a likely association between BP and dengue transmission 


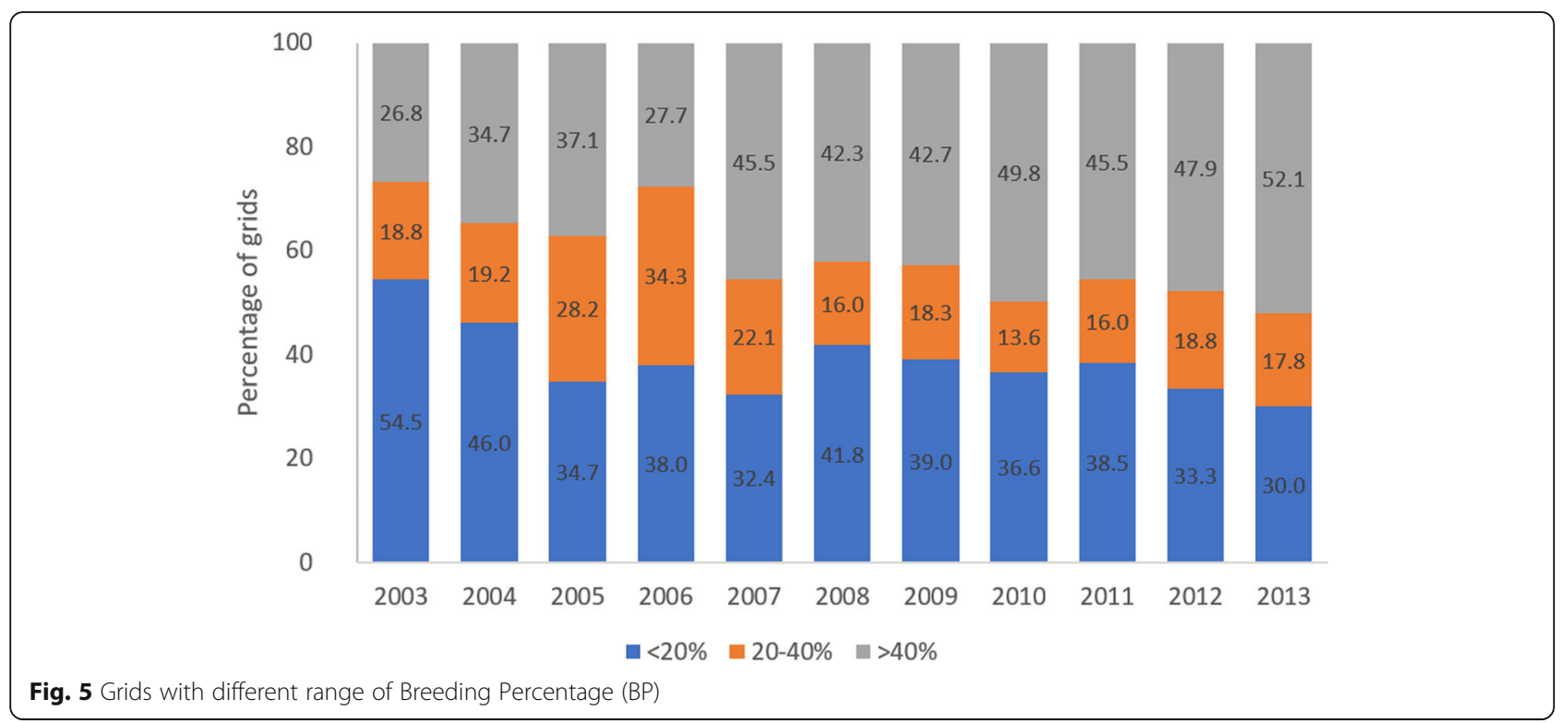

The annual median BP has a strong significant positive correlation with total population (Spearman's correlation: $\left.r_{(11)}=0.893, P<0.0001\right)$ and the number of residential dwelling units (Spearman's correlation: $r_{(11)}=0.875$, $P<0.0001$ ) (Fig. 8).

\section{Discussion}

Vector indices are key risk factors in dengue transmission. Commonly used indices are house index, container index and Breteau index [1]. Globally these indices have been used for risk assessment and early warning for dengue epidemics $[44,45]$. However, there is little solid evidence to quantify the relationship between these indices and dengue transmission [46]. In Singapore, the House
Index has been used by authorities for dengue risk assessment since 1960s and it has been brought down and maintained at low levels by NEA's intensive vector control programme [14]. In spite of the consistent low HI, Singapore remains susceptible to dengue outbreaks, suggesting that the low $\mathrm{HI}$ is no longer sensitive for risk assessment. It highlights the need for a new vector index that accounts for the spatial heterogeneity of dengue transmission risk.

This study introduced a new entomological index, the Ae. aegypti Breeding Percentage (BP), presented its association with case burden, and proposed thresholds for its applications as a risk indicator for dengue control interventions. Absolute number of reported cases was used to

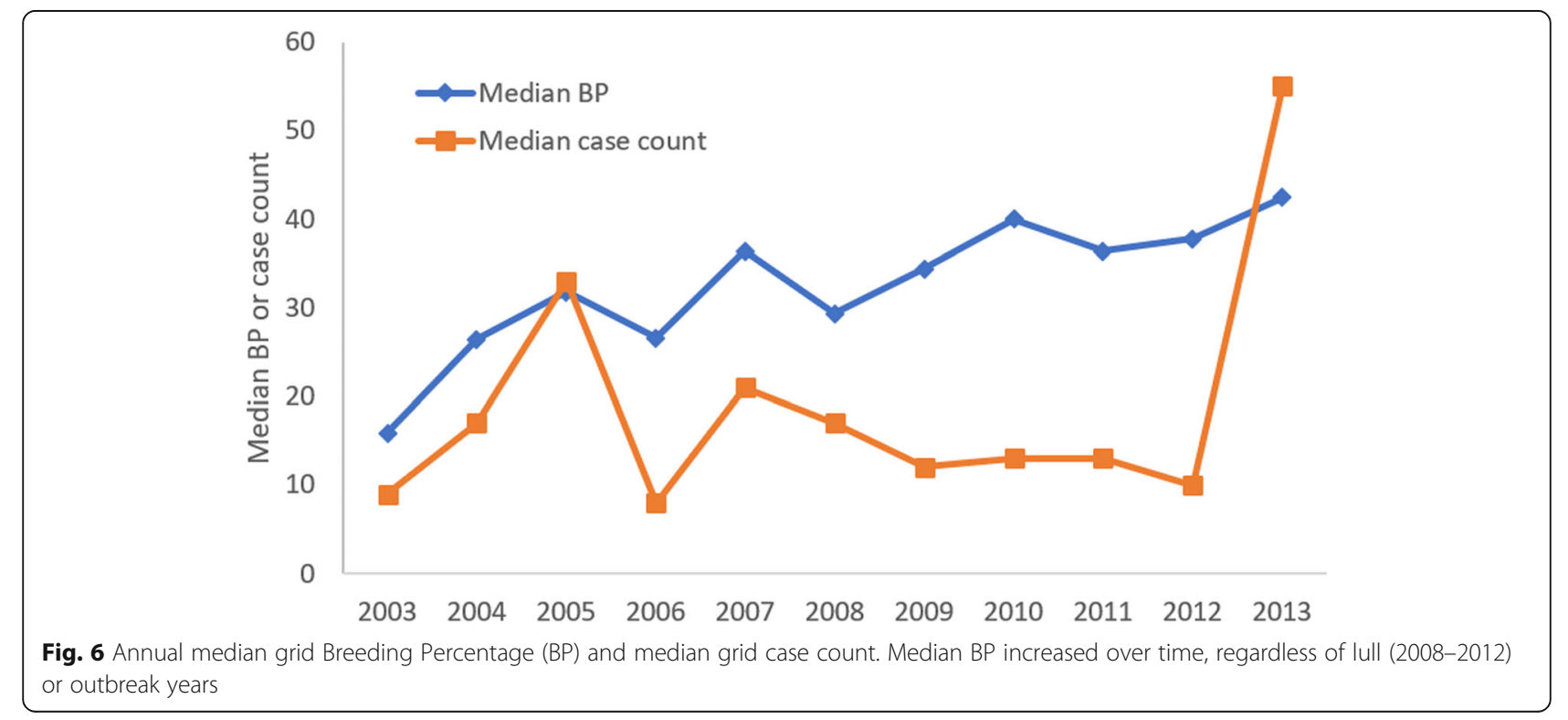




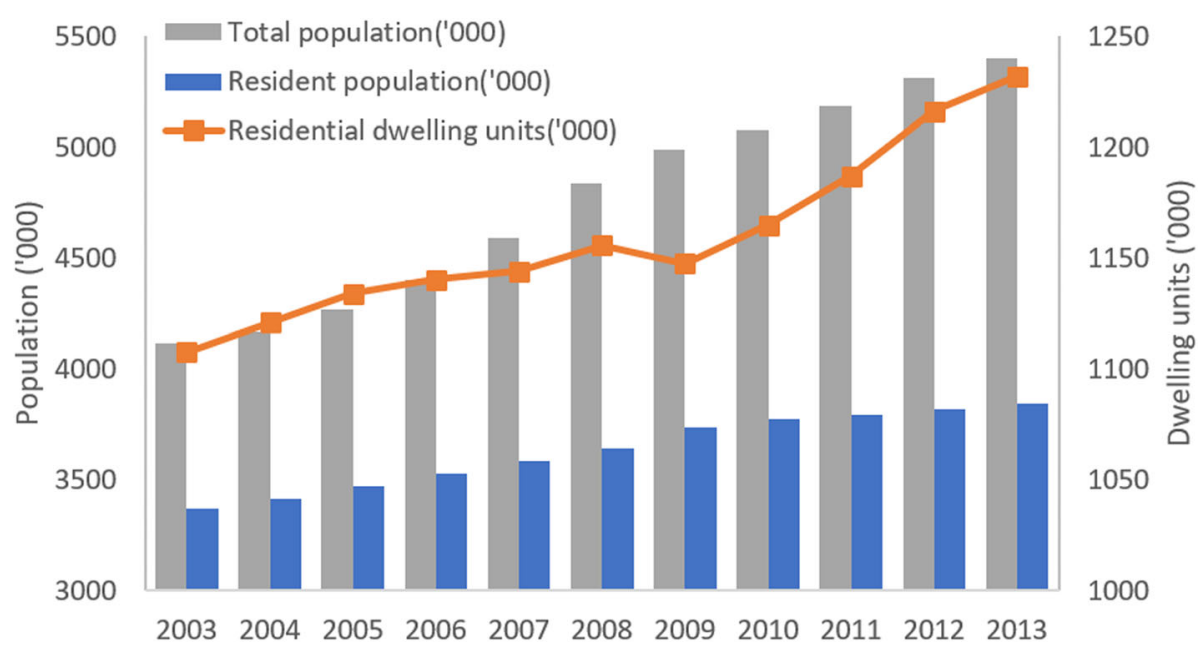

Fig. 7 Population and residential dwelling units in Singapore from 2003 to 2013

measure case burden instead of incidence rate because control interventions are directly related to case count.

Aedes aegypti BP, reported in the present study was developed using the existing Aedes breeding data obtained through the routine vector surveillance programme in Singapore. As routine larval collection efforts are not uniform spatially as well as temporally, using the absolute data for risk assessment would be biased. The development of Ae. aegypti BP takes into consideration the ubiquitous presence of Ae. albopictus and normalizes the data with the total Aedes breeding sites, which comprises of $A e$. aegypti and Ae. albopictus breeding sites. When compared with the traditional Aedes HI, which is reported only on a national level, the main strength of BP is its higher resolution and usefulness for spatial analyses of dengue transmission. We have shown that on a yearly basis, areas with higher BP tend to have higher case count based on historical data. Therefore, BP can be used as a risk factor in spatial risk mapping of dengue transmission together with other factors.

The new index has also demonstrated the expansion of Ae. aegypti into new territories from 2003 to 2013. The strong correlation between annual median BP and annual total population and residential dwelling units suggests the expansion might be a result of rapid population growth and urbanization. However, more thorough analysis is needed to pinpoint the causes conclusively. These new territories have become highly vulnerable to dengue fever outbreaks, probably contributed by the minimal prior exposure of inhabitants to DENV. The low level of immunity to DENV has been demonstrated by our seroprevalence studies [47].

Cases in areas where Ae. aegypti was absent occurred as isolated cases throughout the years, with no evidence of temporal and spatial link among these cases. This could be due to cases acquiring dengue outside of home
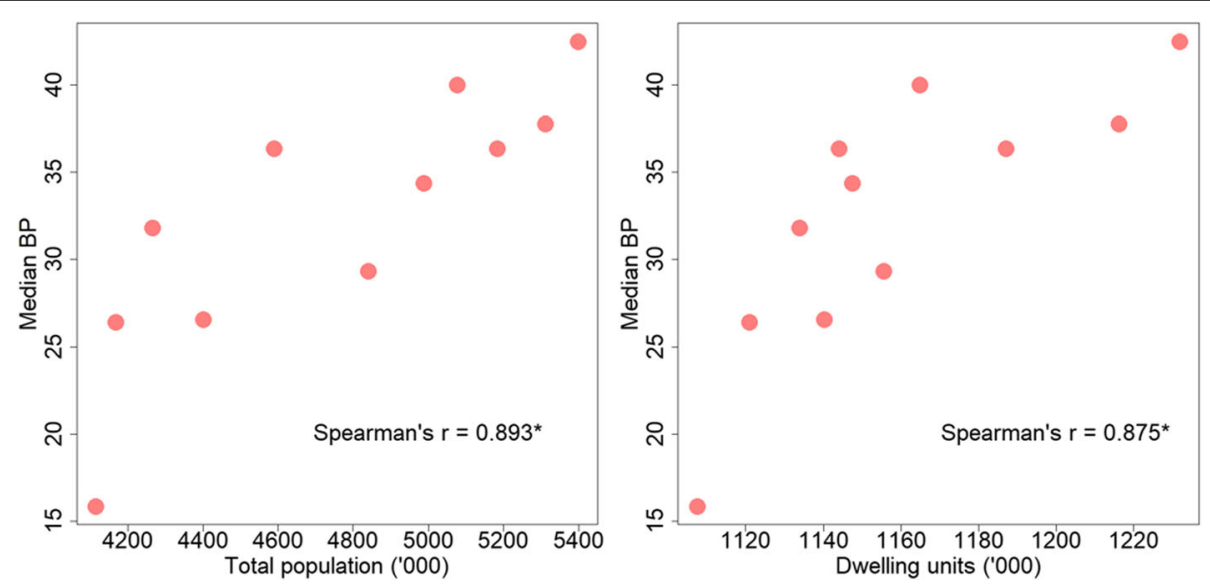

Fig. 8 Relationship between Median Breeding Percentage (BP) with total population and number of residential dwelling units, assessed by Spearman's correlation. Asterisks indicate statistical significance 
and workplace (where cases were tagged to), which is not unexpected considering the dynamic human movement in a city. Alternatively, these small number of cases could be due to the low transmission of Ae. albopictus, which is recognised as a secondary vector of dengue globally.

Thresholds were derived with respect to Singapore's dengue situation and vector population, to ease the application of BP in guiding decisions on the ground to allocate vector control resources. In areas where $A e$. aegypti is entrenched (BP $\geq 20 \%$ ), more resources should be assigned for intensive vector control. In areas where Ae. aegypti has recently infiltrated (BP $<20 \%)$, the focus could aim at elimination of the vector. In more recent years, BP at the national level has been incorporated into a dengue forecast model, which has been useful in predicting dengue trends at least 3 months ahead [48]. BP of each grid has also been incorporated into a spatial risk map which is developed annually, to guide prioritization of resources [49]. These are part of a general effort to stratify risk of dengue temporally and spatially [50].

In view of how chikungunya has adapted to Ae. albopictus, and the increasing number of reports of Ae. albopictus-driven dengue outbreaks internationally, the epidemiology could be dynamic and demands close monitoring to ensure that any change in vector status is detected $[51,52]$.

\section{Conclusions}

With the disease burden of dengue fever increasing across the world, a similar approach would be applicable to other dengue endemic areas, where Ae. albopictus is prevalent, e.g. most of tropical and sub-tropical Asia. The Ae. aegypti BP could be recommended as an indicator for decision making in vector control efforts. It can also be used to monitor the geographical expansion of Ae. aegypti.

\section{Additional file}

Additional file 1: Figure S1. Empirical variogram (circle) and modelled spherical variogram (line) for the residuals of the Breeding Percentage. (TIF $245 \mathrm{~kb})$

\section{Abbreviations}

Bl: Breteau index; BP: Breeding percentage; Cl: Container index; DENV: Dengue virus; GIS: Geographical information system; HI: House index; $\mathrm{MOH}$ : Ministry of Health; NEA: National Environment Agency; WHO: World Health Organization

\section{Acknowledgements}

The authors would like to thank the following people who have contributed to the project; staff of Environmental Public Health Operations involved in mosquito larval surveys and operations, Lam Sai Gek and the Entomologica Taxonomy team for identification of mosquito larval samples, Quek Hui Leng, Chong Chee Seng, Christina Liew, Tan Han Kiat, David Lee, Yew Boon Keat and Tan Cheong Huat for technical discussions and assistance.

\section{Funding}

This study was funded by Singapore's Ministry of Environment and Water Resources "Environment Initiative Fund".

\section{Availability of data and materials}

The datasets generated and/or analysed during the current study are not publicly available due to patient confidentiality but are available from the corresponding author upon reasonable request.

\section{Authors' contributions}

LCN conceptualized, designed and guided the study. JO and XL carried out the study. JO drafted the manuscript. LCN, DH and GY edited the manuscript, and revised it critically. JR handled and processed the data and contributed to the acquisition and cleaning of data. All authors read and approved the final manuscript.

\section{Ethics approval and consent to participate}

The permission to use these data was approved by the Ministry of Health, Singapore and National Environment Agency, Singapore.

\section{Consent for publication}

Not applicable.

\section{Competing interests}

The authors declare that they have no competing interests.

\section{Publisher's Note}

Springer Nature remains neutral with regard to jurisdictional claims in published maps and institutional affiliations.

\section{Author details}

${ }^{1}$ Environmental Health Institute, National Environment Agency, Singapore, Singapore. ${ }^{2}$ Environmental Public Health Operations, National Environment Agency, Singapore, Singapore. ${ }^{3}$ School of Biological Sciences, Nanyang

Technological University, Singapore, Singapore.

Received: 9 August 2018 Accepted: 27 December 2018

Published online: 08 January 2019

\section{References}

1. WHO. Dengue Guidelines for Diagnosis, Treatment, Prevention and Control. New edition. Geneva: World Health Organization; 2009.

2. Gubler DJ. Dengue and dengue hemorrhagic fever. Clin Microbiol Rev. 1998;11:480-96.

3. Chan KL, Ng SK, Chew LM. The 1973 dengue haemorrhagic fever outbreak in Singapore and its control. Singapore Med J. 1977;18:81-93.

4. Goh K, Ng S, Chan Y, Lim S, Chua E. Epidemiological aspects of an outbreak of dengue fever/dengue haemorrhagic fever in Singapore. Southeast Asian J Trop Med Public Health. 1987;18:295-302.

5. Hii YL, Zhu H, Ng N, Ng LC, Rocklöv J. Forecast of dengue incidence using temperature and rainfall. PLoS Negl Trop Dis. 2012;6:e1908.

6. La Ruche $G$, Souarès $Y$, Armengaud $A$, Peloux-Petiot $F$, Delaunay $P$, Desprès $P$, et al. First two autochthonous dengue virus infections in metropolitan France, September 2010. Euro Surveill. 2010;15:19676.

7. Gratz N. Critical review of the vector status of Aedes albopictus. Med Vet Entomol. 2004:18:215-27.

8. Issack MI, Pursem VN, Barkham TM, Ng L-C, Inoue M, Manraj SS Reemergence of dengue in Mauritius. Emerg Infect Dis. 2010;16:716-8.

9. Hapuarachchi HC, Koo C, Rajarethinam J, Chong C-S, Lin C, Yap G, et al. Epidemic resurgence of dengue fever in Singapore in 2013-2014: a virological and entomological perspective. BMC Infect Dis. 2016;16:300.

10. Koh BK, Ng LC, Kita Y, Tang CS, Ang LW, Wong KY, et al. The 2005 dengue epidemic in Singapore: epidemiology prevention and control. Ann Acad Med Singapore. 2008;37:538-45.

11. Lee C, Vythilingam I, Chong C-S, Razak MAA, Tan C-H, Liew C, et al. Gravitraps for management of dengue clusters in Singapore. Am J Trop Med Hyg. 2013:88:888-92.

12. Vikram K, Nagpal BN, Pande V, Srivastava A, Saxena R, Singh H, et al. Detection of dengue virus in individual Aedes aegypti mosquitoes in Delhi, India. J Vector Borne Dis. 2015;52:129-33. 
13. Khan E, Hasan R. Dengue infection in Asia; a regional concern. J Postgrad Med Inst. 2011;26:1-6.

14. Ooi E-E, Goh K-T, Gubler DJ. Dengue prevention and 35 years of vector control in Singapore. Emerg Infect Dis. 2006;12:887-93.

15. Ministry of Health of Singapore. Communicable Diseases Surveillance in Singapore 2003. https://www.moh.gov.sg/resources-statistics/reports/thecommunicable-disease-surveillance-in-singapore-2003. Accessed 8 Oct 2017.

16. Ministry of Health of Singapore. Communicable Diseases Surveillance in Singapore 2005. https://www.moh.gov.sg/resources-statistics/reports/ communicable-diseases-surveillance-in-singapore-2005. Accessed 8 Oct 2017.

17. Ministry of Health of Singapore. Communicable Diseases Surveillance in Singapore 2007. https://www.moh.gov.sg/resources-statistics/reports/ communicable-diseases-surveillance-in-singapore-2007. Accessed 8 Oct 2017.

18. Lee K-S, Lai Y-L, Lo S, Barkham T, Aw P, Ooi P-L, et al. Dengue virus surveillance for early warning, Singapore. Emerg Infect Dis. 2010;16:847-9.

19. Singapore Department of Statistics. Population trends 2014. http://www. singstat. gov. sg/publications/publications-and-papers/population-andpopulation-structure/population_trends. Accessed 08 Oct 2017.

20. Singapore Department of Statistics. Yearbook of Statistics, Singapore Department of Statistics, Ministry of Trade \& Industry, Republic of Singapore: Singapore; 2014.

21. Cornelius-Takahama V. Paya Lebar Airport. 1998. http://eresources.nlb.gov. sg/infopedia/articles/SIP_130_2005-01-22.html. Accessed 08 Oct 2017.

22. Low S-L, Lam S, Wong W-Y, Teo D, Ng L-C, Tan L-K. Dengue seroprevalence of healthy adults in Singapore: serosurvey among blood donors, 2009. Am J Trop Med Hyg. 2015;93:40-5

23. Wilder-Smith A, Foo W, Earnest A, Sremulanathan S, Paton NI. Seroepidemiology of dengue in the adult population of Singapore. Trop Med Int Health. 2004;9:305-8.

24. Yew YW, Ye T, Ang LW, Ng LC, Yap G, James L, et al. Seroepidemiology of dengue virus infection among adults in Singapore. Ann Acad Med Singapore. 2009;38:667-75.

25. Chang MS, Christophel EM, Gopinath D, Abdur RM. Challenges and future perspective for denque vector control in the Western Pacific Region. Western Pac Surveill Response J. 2011;2:9-16.

26. Barrera R, Amador M, Diaz A, Smith J, Munoz-Jordan J, Rosario Y. Unusual productivity of Aedes aegypti in septic tanks and its implications for dengue control. Med Vet Entomol. 2008;22:62-9.

27. Caputo B, lenco A, Cianci D, Pombi M, Petrarca V, Baseggio A, et al. The "auto-dissemination" approach: a novel concept to fight Aedes albopictus in urban areas. PLoS Negl Trop Dis. 2012;6:e1793.

28. Tatem AJ, Rogers DJ, Hay S. Global transport networks and infectious disease spread. Adv Parasitol. 2006;62:293-343.

29. Tatem AJ, Hay SI, Rogers DJ. Global traffic and disease vector dispersal. Proc Natl Acad Sci USA. 2006;103:6242-7.

30. Semenza JC, Sudre B, Miniota J, Rossi M, Hu W, Kossowsky D, et al. International dispersal of dengue through air travel: importation risk for Europe. PLoS Negl Trop Dis. 2014;8:e3278.

31. Tun-Lin W, Kay B, Barnes A, Forsyth S. Critical examination of Aedes aegypti indices: correlations with abundance. Am J Trop Med Hyg. 1996;54:543-7.

32. Focks DAA. Review of Entomological Sampling Methods and Indicators for Dengue vectors. Geneva: World Health Organization; 2004.

33. WHO. Guidelines for Dengue Surveillance and Mosquito Control. Manila: WHO Regional Office for the Western Pacific; 2003

34. Ministry of Health of Singapore. Infectious Diseases Act. https://www.moh. gov.sg/policies-and-legislation/infectious-disease-act. Accessed 08 Oct 2017.

35. Lai Y-L, Chung Y-K, Tan H-C, Yap H-F, Yap G, Ooi E-E, et al. Cost-effective real-time reverse transcriptase PCR (RT-PCR) to screen for dengue virus followed by rapid single-tube multiplex RT-PCR for serotyping of the virus. J Clin Microbiol. 2007:45:935-41.

36. Pok K-Y, Lai Y-L, Sng J, Ng L-C. Evaluation of nonstructural 1 antigen assays for the diagnosis and surveillance of dengue in Singapore. Vector Borne Zoonotic Dis. 2010;10:1009-16.

37. Rattanarithikul R, Harbach RE, Harrison BA, Panthusiri P, Coleman RE, Richardson JH. Illustrated keys to the mosquitoes of Thailand. VI. Tribe Aedini. Southeast Asian J Trop Med Public Health. 2010;41:1-225.

38. Singapore Department of Statistics. Yearbook of Statistics, Singapore. Department of Statistics, Ministry of Trade \& Industry, Republic of Singapore: Singapore; 2009
39. R Development Core Team. R. A language and environment for statistical computing. Vienna: R Foundation for Statistical Computing; 2016. https:// www.R-project.org/

40. Morales M. Sciplot: scientific graphing functions for factorial designs. R package version 2011:1-0.

41. Pebesma E, Bivand RS. Classes and methods for spatial data: The sp Package. R News. 2005;5:9-13.

42. Pebesma EJ. Multivariable geostatistics in S: The gstat package. Comput Geosci. 2004;30:683-91.

43. Robin X, Turck N, Hainard A, Tiberti N, Lisacek F, Sanchez J-C, et al. pROC: an open-source package for $\mathrm{R}$ and $\mathrm{S}+$ to analyze and compare ROC curves. BMC Bioinformatics. 2011;12:77

44. Sanchez L, Cortinas J, Pelaez O, Gutierrez H, Concepción D, Van der Stuyft P. Breteau Index threshold levels indicating risk for dengue transmission in areas with low Aedes infestation. Trop Med Int Health. 2010;15:173-5.

45. Sanchez L, Vanlerberghe V, Alfonso L, del Carmen Marquetti M, Guzman $M G$, Bisset J, et al. Aedes aegypti larval indices and risk for dengue epidemics. Emerg Infect Dis. 2006;12:800-6.

46. Bowman $L R$, Runge-Ranzinger $S$, McCall P. Assessing the relationship between vector indices and dengue transmission: a systematic review of the evidence. PLoS Negl Trop Dis. 2014;8:e2848.

47. Ler TS, Ang LW, Yap GSL, Ng LC, Tai JC, James L, et al. Epidemiological characteristics of the 2005 and 2007 dengue epidemics in Singapore similarities and distinctions. Western Pac Surveill Response J. 2011:2:24-9.

48. Shi Y, Liu X, Kok S-Y, Rajarethinam J, Liang S, Yap G, et al. Three-month realtime dengue forecast models: an early warning system for outbreak alerts and policy decision support in Singapore. Environ Health Perspect. 2015; 124:1369-75.

49. Ong J, Liu X, Rajarethinam J, Kok SY, Liang S, Tang CS, et al. Mapping dengue risk in Singapore using Random Forest. PLoS Negl Trop Dis. 2018; 12:e0006587.

50. Ng L, Tan H, Tan L, Chong C, Ho D. Evolving dengue control programme in Singapore. Epidemiol News Bull. 2016:42:11-5.

51. Kek R, Hapuarachchi H, Chung C-Y, Humaidi MB, Razak MAB, Chiang S, et al. Feeding host range of Aedes albopictus (Diptera: Culicidae) demonstrates its opportunistic host-seeking behavior in rural Singapore. J Med Entomol. 2014;51:880-4.

52. Lambrechts L, Scott TW, Gubler DJ. Consequences of the expanding global distribution of Aedes albopictus for denque virus transmission. PLoS Neg Trop Dis. 2010;4:e646.

\section{Ready to submit your research? Choose BMC and benefit from:}

- fast, convenient online submission

- thorough peer review by experienced researchers in your field

- rapid publication on acceptance

- support for research data, including large and complex data types

- gold Open Access which fosters wider collaboration and increased citations

- maximum visibility for your research: over $100 \mathrm{M}$ website views per year

At BMC, research is always in progress.

Learn more biomedcentral.com/submission 